\title{
THE DEVELOPMENT OF SUNSPOT GROUPS
}

\author{
ROBERT F. HOWARD \\ National Solar Observatory, National Optical Astronomy Observatories, ${ }^{*}$ \\ Tucson, AZ 85726
}

\begin{abstract}
It is shown that the growth and decay rates of sunspot groups are both a function of the axial tilt angles of the groups. Both of these rates reach a maximum near the average tilt angle, which is around $+5^{\circ}$, not $0^{\circ}$. (A positive tilt angle represents the situation where the leading spots are equatorward of the following spots). The fact that this peak angle is close to the average tilt and not $0^{\circ}$ suggests that this may be the orientation of the subsurface toroidal flux tube(s) from which the sunspot groups form. In general, spot groups with positive tilt angles show faster average percentage growth rates for greater positive tilt angles. For negative tilt angles (following spots equatorward of leading spots) the situation is quite different. Within certain well-defined ranges of tilt angles the average group percentage area growth rates are quite high and the dispersion in this quantity is large. In other well-defined tilt angle ranges the percentage growth rates are uniformly (relatively) small. The difference between these average growth rates is more than a factor two. The reality of this result can be demonstrated by selecting for analysis various subsets of the data. It may be hypothesized that in general the dispersion measured in group tilt angles results from the random action of large-scale convection on the rising magnetic flux loops. Both growth and decay are facilitated in the case of flux loops that have a minimum twist. The percentage growth and decay rates are affected by the fact that groups with tilt angles near the average value tend to be larger than groups with different tilt angles. The 'quantized' behavior of the percentage growth rates for negative tilt angles is difficult to fit into any rough model of flux emergence. The more or less 'quantized' nature of the growth-tilt angle relationship for negative tilt angles is quite puzzling and may reflect some characteristic of the subsurface flux orientation or development which is at present unknown.
\end{abstract}

\section{INTRODUCTION}

It is generally believed that sunspot groups and plages form by the emergence of one or more loops of magnetic field lines that have risen from some depth,

* Operated by the Association of Universities for Research in Astronomy, Inc., under Cooperative Agreement with the National Science Foundation. 
perhaps near the base of the convection zone (Parker 1987). Some properties of the rising flux, which is assumed to originate from a toroidal source flux tube, may be affected in its buoyant rise to the photosphere (Parker, 1988) by a vertical rotation rate gradient and by large-scale convective motions beneath the surface. These properties include region areas, latitude drifts, and east-west inclination angles (Howard 1991a, 1991b, 1992). Two other region characteristics that may possibly be affected by subsurface conditions are the growth and decay rates of regions. In this study, the Mount Wilson sunspot data set (Howard, Gilman, and Gilman, 1984) is used to examine these rates as a function of the tilt angle of the magnetic axis of the spot group (Howard 1993).

\section{Data and Analysis}

The digitized Mount Wilson data set has been described (Howard, Gilman, and Gilman 1984). The current version of this data set covers the interval 19171985. The individual measurements of umbral area and position are combined into sunspot groups, whose positions are area-weighted. The derivation of tilt angles of the spot groups was discussed earlier (Howard 1991c, 1993).

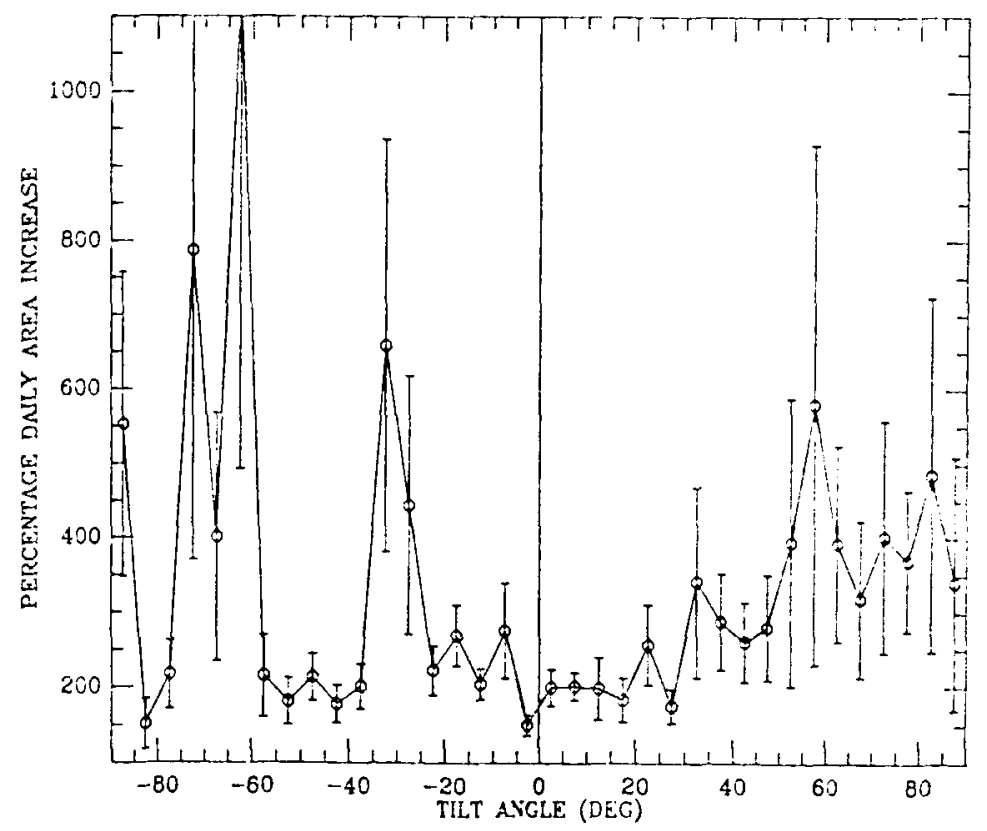

Fig. 1. The daily percentage spot group umbral area increase(for groups whose areas are increasing only) averaged over $5-^{\circ}$ intervals of group tilt angle for the full interval of the data. Note the discrete zones of rapid growth for negative tilt angles.

The determination of groups from the individual spot measures is 
discussed in the earlier paper (Howard, Gilman, and Gilman, 1984). The umbral areas of all the individual sunspots are summed to determine the total group area for each day, corrected for foreshortening. The growth or decay rate is just the difference between these two quantities on two consecutive days.

\section{$\underline{\text { Results }}$}

Plots of the daily umbral area increase or decrease show peaks near the average tilt angle. For decaying groups, the largest decay rates are near the average angle (Howard 1993). A plot of the percentage daily umbral area increase averaged over 5 -deg intervals of tilt angle shows at the average tilt angle a minimum, i.e. the largest (absolute) negative rate (Howard 1993). The reason for this is that the average group size is larger at the average tilt angle than on either side of that quantity (Howard 1991c).

Figure 1 shows the percentage daily umbral area increase averaged over 5-deg intervals in tilt angle. Here the values near the average tilt angle are relatively low and increase somewhat for larger positive tilt angles. For negative tilt angles, values are low for tilt angle ranges of about $0^{\circ}-30^{\circ}, 40^{\circ}-$ $60^{\circ}$, and around $80^{\circ}$. The error bars are significantly larger for the higher values, suggesting a greater dispersion in these ranges. This plot by itself is not very convincing because of the large error bars in these intervals, but similar plots made for the northern and southern hemispheres and for the first and second halves of the data set show quite similar behavior (Howard 1993) suggesting that the effect is real.

\section{Conclusions and Discussion}

It is suggested, and discussed in more detail in the full paper, that the peaks in the growth and decay rates result from the fact that the average tilt angle represents the state of minimum twist of the subsurface flux tube that forms the spot group. Both the growth and decay of sunspots, it is hypothesized, will proceed more rapidly when the twist of the flux tube is at a minimum. This implies that the subsurface source flux tube from which the group forms has the same tilt as the average of groups. The result in Figure 1 may represent complex effects at work beneath the surface to make the growth of spots at certain negative tilt angles more rapid.

\section{REFERENCES}

Howard, R. F. 1991a, Solar Phys., 135, 327.

Howard, R. F. 1991b, Solar Phys., 135, 339.

Howard, R. F. 1991c, Solar Phys., 136, 251.

Howard, R. F. 1992, Solar Phys., 137, 205.

Howard, R. F. 1993, Solar Phys., (in press).

Howard, R. F., Gilman, P. A., and Gilman, P. I. 1984, Ap. J., 283, 373.

Parker, E. N. 1987, Solar Phys., 110, 11.

Parker, E. N. 1988, Ap. J., 325, 880. 